\title{
Role of interferon regulatory factor-1 in lipopolysaccharide-induced mitochondrial damage and oxidative stress responses in macrophages
}

\author{
SONG-YUN DENG ${ }^{1}$, LE-MENG ZHANG ${ }^{1}$, YU-HANG AI ${ }^{1}$, PIN-HUA PAN ${ }^{2}$, SHUANG-PING ZHAO $^{1}$, \\ XIAO-LI SU ${ }^{2}$, DONG-DONG WU ${ }^{2}$, HONG-YI TAN ${ }^{2}$, LI-NA ZHANG ${ }^{1}$ and ALLAN TSUNG ${ }^{3}$ \\ Departments of ${ }^{1}$ Intensive Care Unit, ${ }^{2}$ Respiratory Medicine, Xiangya Hospital, Central South University, Changsha, \\ Hunan 410008, P.R. China; ${ }^{3}$ Department of Surgery, University of Pittsburgh, Pittsburgh, PA 15213, USA
}

Received May 9, 2016; Accepted August 4, 2017

DOI: $10.3892 / \mathrm{ijmm} .2017 .3110$

\begin{abstract}
Sepsis causes many early deaths; both macrophage mitochondrial damage and oxidative stress responses are key factors in its pathogenesis. Although the exact mechanisms responsible for sepsis-induced mitochondrial damage are unknown, the nuclear transcription factor, interferon regulatory factor-1 (IRF-1) has been reported to cause mitochondrial damage in several diseases. Previously, we reported that in addition to promoting systemic inflammation, IRF-1 promoted the apoptosis of and inhibited autophagy in macrophages. In the present study, we hypothesized that lipopolysaccharide (LPS)-induced IRF-1 activation in macrophages may promote mitochondrial damage and oxidative stress. In vitro, LPS was found to promote IRF-1 activation, reactive oxygen species (ROS) production, adenosine triphosphate (ATP) depletion, superoxide dismutase (SOD) consumption, malondialdehyde (MDA) accumulation and mitochondrial depolarization in macrophages in a time- and dose-dependent manner. These effects were abrogated in cells in which IRF-1 was knocked down. Furthermore, IRF-1 overexpression increased LPS-induced oxidative stress responses and mitochondrial damage. In vivo, peritoneal macrophages obtained from IRF-1 knockout (KO) mice produced less ROS and had less mitochondrial depolarization and damage following
\end{abstract}

Correspondence to: Professor Yu-Hang Ai or Dr Le-Meng Zhang, Department of Intensive Care Unit, Xiangya Hospital, Central South University, Changsha, Hunan 410008, P.R. China

E-mail: ayhicu1978@126.com

E-mail: 497730787@qq.com

Abbreviations: LPS, lipopolysaccharide; IRF-1, interferon regulatory factor-1; mtDNA, mitochondrial DNA; WT, wild-type; KO, knockout; KD, knockdown; PBS, phosphate-buffered saline; IP, intraperitoneal; ROS, reactive oxygen species; SOD, superoxide dismutase; MDA, malondialdehyde; ATP, adenosine triphosphate; TEM, transmission electron microscope

Key words: interferon regulatory factor-1, endotoxemia, macrophage, oxidative stress response, mitochondrial damage, mitochondrial DNA the administration of LPS, when compared to their wildtype (WT) counterparts. In addition, IRF-1 KO mice exhibited a decreased release of mitochondrial DNA (mtDNA) following the administration of LPS. Thus, IRF-1 may be a critical factor in augmenting LPS-induced oxidative stress and mitochondrial damage in macrophages.

\section{Introduction}

Sepsis, caused by severe infections, burns and trauma, remains a leading cause of mortality worldwide. In the US, the mortality rate due to sepsis is as high as $50 \%$ and is increasing at a rate of $1.5 \%$ per year. Thus, approximately 200,000 individuals die from sepsis each year, and health care costs associated with this exceed $\$ 16$ billion annually $(1,2)$. In China, severe sepsis has a mortality rate of $40.7 \%$ during the first 28 days, and health care costs are at 90,000 Yuan/patient (3). Accordingly, further studies are required in order to obtain a better understanding of the pathophysiology of sepsis and available clinical treatment options.

Lipopolysaccharide (LPS)-induced endotoxemia mimics many features of septic shock (4), including the persistent activation of systemic inflammatory responses, immune suppression, coagulation disorders and mitochondrial damage (2). Macrophage mitochondrial structural damage and consequent oxidative stress responses are associated with apoptosis, energy metabolism fluctuations and systemic inflammatory responses; these all contribute to septic shock and multiple organ failure (5).

Macrophages are the first line of defense against pathogens and are important regulators of innate and adaptive immune responses (6). Macrophage mitochondrial dysfunction is responsible for changes in energy metabolism, the intrinsic apoptotic pathway, oxidative stress and systemic inflammatory responses. The association between the degree of macrophage mitochondrial dysfunction and deleterious outcomes has been documented; inflammatory factors, free radicals, calcium overload, adenosine triphosphate (ATP)-sensitive potassium channel perturbations and respiratory chain complex damage are responsible for the noted mitochondrial damage during endotoxemia (7). However, the upstream signaling that mediates 
these molecular events leading to mitochondrial dysfunction is poorly understood.

Interferon regulatory factor-1 (IRF-1) is an important nuclear transcription factor with various biological functions, including the promotion of systemic inflammatory responses and apoptosis, the regulation of the development and differentiation of immune cells, and the inhibition of cell proliferation (8). IRF-1 was first recognized for regulating type I interferon production, and it has been indicated that IRF-1 can promote the transcription of diverse inflammatory factors, such as interleukin (IL)-6, IL-12 and IL-18 (9). IRF-1 has also been shown to be involved in LPS-induced multiple organ damage and death. IRF-1 can also promote the release of numerous inflammatory factors, including the early inflammatory cytokines tumor necrosis factor- $\alpha$ (TNF- $\alpha$ ) and interferon- $\gamma$ (IFN- $\gamma$ ), as well as the late inflammatory danger signal, high mobility group box 1 (HMGB1) $(10,11)$. We have previously demonstrated that IRF-1 inhibited the autophagy responses of immune cells, such as macrophages and splenocytes $(12,13)$. Since autophagy is important for mitochondrial quality control and the maintenance of mitochondrial homeostasis (14-16), we hypothesized that IRF-1 activation may disrupt mitochondrial homeostasis.

IRF-1 activation may also directly damage mitochondria in both primary cells and cancer cell lines. Mitochondrial membrane permeability increases and cytochrome $c$ is released in gastric cancer cells overexpressing IRF-1 (17). IRF-1 gene knockout can alleviate LPS/D-GalN-induced hepatocellular mitochondrial damage and reduce intrinsic apoptosis (18). However, whether IRF-1 participates in LPS-induced macrophage mitochondrial damage and oxidative stress remains unknown. We previously reported that IRF-1-mediated immune cell apoptosis and autophagy plays an important role in LPS-induced multiple organ failure and death $(12,13)$. In this study, we report a novel role for IRF-1 in LPS-induced oxidative stress responses and mitochondrial structural damage in macrophages.

\section{Materials and methods}

Animals. IRF-1 knockout $(\mathrm{KO} ; \mathrm{n}=48)$ and matched C57BL/6J wild-type (WT; $\mathrm{n}=48$ ) (8-10 weeks of age, male, 25-30 g) mice were purchased from the Jackson Laboratory (Bar Harbor, ME, USA). Animals were maintained in a specific pathogen-free, laminar-flow housing apparatus under controlled temperature, humidity and a $12 \mathrm{~h}$ light/dark regimen. The Animal Care and Use Committee of the Central South University approved all animal protocols. All experiments were conducted in accordance with the National Institutes of Health Guidelines for the Care and Use of Laboratory Animals.

In vivo experimental design. The mice were randomly assigned to 4 groups ( $\mathrm{n}=8$ in each) as follows: WT + phosphate-buffered saline (PBS), WT + LPS, IRF-1 KO + PBS and IRF-1 KO + LPS groups. The mice in the LPS groups were administered LPS (Escherichia coli 0111:B4) (Sigma-Aldrich, St. Louis, MO, USA) (20 mg/kg, i.p.). The mice in the PBS groups received treatment with sterilized PBS. At $16 \mathrm{~h}$ after the administration of PBS or LPS, the mice were anesthetized with chloral hydrate $(400 \mathrm{mg} / \mathrm{kg})$. Blood samples and peritoneal macrophages were collected and the mice were sacrificed.
Isolation of peritoneal macrophages. Mouse abdomens were washed with $70 \%$ ethanol and a lateral incision was made with scissors along the bottom midline of the peritoneum. With forceps, abdominal skin was retracted to expose the transparent peritoneal skin. Subsequently, $5 \mathrm{ml}$ syringes were attached to $20 \mathrm{G}$ needles and $3 \mathrm{ml}$ of cold RPMI-1640 cell culture medium was injected into the peritoneal cavity of each mouse. The peritoneal cavity was massaged and peritoneal fluid was carefully aspirated and placed into $15 \mathrm{ml}$ centrifuge tubes (19). This was repeated for 3 treatments and the samples were centrifuged for $10 \mathrm{~min}$ at $300 \mathrm{x} \mathrm{g}$. The supernatant was discarded and the cell pellet was resuspended in RPMI-1640 cell culture medium.

Cell culture and treatment. Murine monocyte/macrophage-like cells (RAW264.7; $10^{6}$ cells; American Type Culture Collection, Manassas, VA, USA) were cultured in RPMI-1640 cell culture medium, supplemented with $10 \% \mathrm{FBS}, 50 \mathrm{U} / \mathrm{ml}$ penicillin, and $50 \mu \mathrm{g} / \mathrm{ml}$ streptomycin (Gibco, Grand Island, NY, USA) in $6 \mathrm{~cm}$ culture plates. The cells were stimulated with LPS at various concentrations $(0,10,100,500$ and $1,000 \mathrm{ng} / \mathrm{ml})$ for different periods of time $(0,1,2,4,8$ and $16 \mathrm{~h})$.

Stable transfection of RAW264.7 cells for overexpression or knockdown of IRF-1. Lentiviral constructs expressing shRNA directed against mouse IRF-1 mRNA were custom-manufactured (Hanyin, Shanghai, China). shRNA sequences were as follows: IRF-1 shRNA, 5'-GCACTAAATGAGTCCTAT TCC-3', and non-specific shRNA, 5'-TTCTCCGAACGTGTCA CGT-3'. The lentiviral constructs expressing mouse IRF-1 were also manufactured by the same vendor (Hanyin). IRF-1 cDNA was obtained from a commercially available source (National Center for Biotechnology Information, Bethesda, MD, USA). All lentiviral vectors were purified to a titer of $1 \times 10^{9} \mathrm{TU} / \mathrm{ml}$. The constructs were transfected into the cells at a multiplicity of infection (MOI) of 20:1 using $5 \mu \mathrm{g} / \mathrm{ml}$ Polybrene in RPMI-1640 cell culture medium. At $4 \mathrm{~h}$ after transfection, the medium was exchanged, and the cells were cultured for an additional $48 \mathrm{~h}$. The effects of IRF-1 knockdown and overexpression were measured by western blot analysis.

Nuclear protein extraction and western blot analysis. The collected cells were centrifuged at $300 \mathrm{x}$ g for $5 \mathrm{~min}$ at $4^{\circ} \mathrm{C}$ The pellets were then resuspended on ice with cytoplasmic extraction reagent (Vazyme, Nanjing, China) combined with protease inhibitor and mixed for $10 \mathrm{~min}$, and centrifuged at $16,000 \mathrm{x} \mathrm{g}$ for $5 \mathrm{~min}$ at $4^{\circ} \mathrm{C}$ to obtain protein. For nuclear protein isolation, the cell pellet was lysed with nuclear extraction reagent (Vazyme) plus protease inhibitor mix.

Protein concentrations were determined by a BCA protein assay kit (Beyotime, Haimen, China), and $40 \mathrm{mg}$ protein per sample were mixed with sample loading buffer and boiled at $96^{\circ} \mathrm{C}$ for $5 \mathrm{~min}$. The samples were then separated using sodium dodecyl sulfate-polyacrylamide gel electrophoresis (SDS-PAGE) and transferred onto polyvinylidene fluoride (PVDF) membranes (Millipore, Billerica, MA, USA). After blocking with 5\% non-fat milk in $10 \mathrm{mM}$ Tris-buffered saline ( $\mathrm{pH} 7.5)$ with $0.1 \%$ Tween-20 (TBS-T) for $1 \mathrm{~h}$ at room temperature, the membranes were incubated overnight at $4^{\circ} \mathrm{C}$ with primary antibodies against rabbit monoclonal antibody of IRF-1 (1:1,000; Cat. no. 8478) and Histone H3 (1:2,000; Cat. no. 4499) (Cell 
Signaling Technology, Beverly, MA, USA). After 3 washes in TBS-T, the membranes were incubated with secondary antibody conjugated with horseradish peroxidase (HRP) at room temperature for $1 \mathrm{~h}$. After 3 washes, the blots were developed with electrochemiluminescence (ECL) (Beyotime) and visualized with a ChemiDoc MP imaging system (Bio-Rad Laboratories, Berkeley, CA, USA). Band density was quantified using ImageJ software version 1.49 (National Institutes of Health, Bethesda, MD, USA).

Detection of mitochondrial transmembrane potential and reactive oxygen species (ROS). Mitochondrial transmembrane potential was assessed using the sensitive fluorescent probe, 5,5',6,6'-tetrachloro-1,1',3,3'-tetraethyl-benzimidazolylcarbocyanine iodide (JC-1) (Invitrogen, Carlsbad, CA, USA). Red emission from the dye is attributed to a potential-dependent aggregation of JC-1 in the mitochondria. Green fluorescence reflects the monomeric form of JC-1, appearing in the cytoplasm after mitochondrial membrane depolarization. The cells were seeded at $3 \times 10^{5}$ cells/well into 6-well plates and incubated overnight at $37^{\circ} \mathrm{C}$ in a carbon dioxide incubator. The cells were then washed with PBS and trypsinized, resuspended in $2 \mathrm{mM}$ of JC-1 at $37^{\circ} \mathrm{C}$ for $30 \mathrm{~min}$. The cells were washed 3 times with PBS, resuspended in $500 \mu \mathrm{l} \mathrm{PBS}$, and then analyzed immediately with a BD FACSCanto II flow cytometer (BD Biosciences, San Jose, CA, USA).

The collected cells were resuspended in $5 \mu \mathrm{M}$ 2,7-dichlorofluorescin diacetate (DCFH-DA) (Sigma-Aldrich, St. Louis, MO, USA) staining solution at $37^{\circ} \mathrm{C}$ for $25 \mathrm{~min}$. Cell suspensions were mixed by inversion every $5 \mathrm{~min}$, making full contact with the probe. The cells were then washed 3 times with PBS to remove DCFH-DA that did not enter the cell. Fluorescence intensity was measured by flow cytometry, as previously described (20).

Transmission electron microscopy (TEM). The cells were fixed in cold $2.5 \%$ glutaraldehyde in PBS and post-fixed in $1 \%$ osmium tetroxide with $0.1 \%$ potassium ferricyanide, dehydrated through a graded series of ethanol (30-90\%) and embedded in Epon. The Epon blocks were then sectioned. Ultrathin sections $(65 \mathrm{~nm})$ were stained with $2 \%$ uranyl acetate and Reynolds lead citrate. The sections were examined under a H-7500 TEM (Hitachi, Tokyo, Japan) by an electron microscopy specialist from the Electron Microscopy Laboratory of Central South University.

Biochemical analysis of intracellular ATP, superoxide dismutase (SOD) and malondialdehyde (MDA). The cells were seeded at $3 \times 10^{5}$ cells/well into 6 -well plates and incubated overnight at $37^{\circ} \mathrm{C}$ in a carbon dioxide incubator and stimulated with LPS (500 ng/ml, $16 \mathrm{~h}$ ). The cells were then washed with PBS and trypsinized, resuspended in PBS and lysed on ice by sonication (Sonics Inc., Qingdao, China) at $100 \%$ amplitude (10x, $30 \mathrm{sec}$ each with $30 \mathrm{sec}$ intervals). Biochemical analysis of MDA, SOD and ATP was carried out using respective kits (Nanjing Jiancheng Bioengineering Institute, Nanjing, China) following the manufacture's instructions.

Purification of circulating DNA and quantitative PCR ( $q P C R)$. Circulating DNA was isolated from plasma with an EZNA circulating DNA kit (Omega Bio-Tek, Norcross, GA, USA) following manufacturer's instructions. The concentrations of DNA were assessed using a NanoDrop 2000 spectrophotometer (Thermo Fisher Scientific, Waltham, MA, USA). Ratios of A260/A280 ranged from 1.9-2.1.

Relative mitochondrial DNA was measured by qPCR using SYBR-Green PCR Master Mix (GeneCopoeia, Guangzhou, China). Reactions were carried out in a $20 \mu \mathrm{l}$ final volume, containing $0.2 \mu \mathrm{M}$ of each forward and reverse primer, $20 \mathrm{ng}$ DNA sample, and $10 \mu \mathrm{l}$ SYBR-Green PCR Master Mix. Amplification was performed with an Applied Biosystems 7300 Real-Time PCR machine (Life Technologies, Carlsbad, CA, USA) under the thermal profile of $95^{\circ} \mathrm{C}$ for $10 \mathrm{~min}$ followed by 40 cycles at $95^{\circ} \mathrm{C}$ for $15 \mathrm{sec}, 60^{\circ} \mathrm{C}$ for $20 \mathrm{sec}$ and $72^{\circ} \mathrm{C}$ for $20 \mathrm{sec}$. Samples that produced no PCR products after 40 cycles were considered 'undetectable'. The threshold cycle $(\mathrm{Ct})$ was obtained from triplicate samples and averaged.

mtDNA copies were calculated based on the ' $\Delta \Delta \mathrm{Ct}$ ' method, using the equation $\mathrm{R}$ (ratio) $=2^{-\Delta \Delta \mathrm{Ct}}$, cytochrome $c$ oxidase 1 (mtCOI) DNA standardized by the housekeeping gene, 18s RNA (encoding 18S ribosomal RNA). The following primers were used: mtCOI forward, 5'-GCCCCAGATATAG CATTCCC-3'; and reverse, 5'-GTTCATCCTGTTCCTGC TCC-3' and 18S RNA forward, 5'-TAGAGGGACAAGTGGCG TTC-3'; and reverse, 5'-CGCTGAGCCAGTCAGTGT-3' (21). Sequence data were analyzed using Blast nucleic acid database searches from the National Centre for Biotechnology Information, and had no significant homology with DNA found in any bacterial species.

Statistical analyses. All data are expressed as the means \pm SEM. Significant differences within groups were analyzed with repeated measures ANOVA followed by an LSD test and significant differences between groups were assessed with one-way ANOVA (a value of $\mathrm{p}<0.05$ was considered to indicate a statistically significant difference). All calculations and statistical analyses were performed using SPSS software for Windows (version 17.0).

\section{Results}

LPS induces IRF-1 activation in a time- and dose-dependent manner in RAW264.7 cells. IRF-1 is a key regulator of immunity and plays an important role in the progression of endotoxemia (9). In order to investigate the expression pattern of IRF-1, the RAW264.7 cells were stimulated with LPS at various concentrations and durations as described in the Materials and methods. Western blot analysis confirmed that IRF-1 nuclear protein peaked at $8 \mathrm{~h}$ after LPS stimulation (Fig. 1A), and LPS induced the peak activation of nuclear IRF-1 at $500 \mathrm{ng} / \mathrm{ml}$ (Fig. 1B). Thus, LPS induced IRF-1 activation in the RAW264.7 cells in a time- and dose-dependent manner.

LPS induces mitochondrial damage and ROS production was time- and dose-dependent. It has been clearly described that mitochondrial damage and ROS production are very important in the pathophysiology of sepsis (22). In this study, we stimulated the RAW264.7 cells with LPS (10-500 ng/ $\mathrm{ml}$ ) for 2-16 h. Mitochondrial membrane depolarization and ROS production were measured by JC-1 or DCFH-DA flow cytometry. Mitochondrial depolarization increased after LPS 

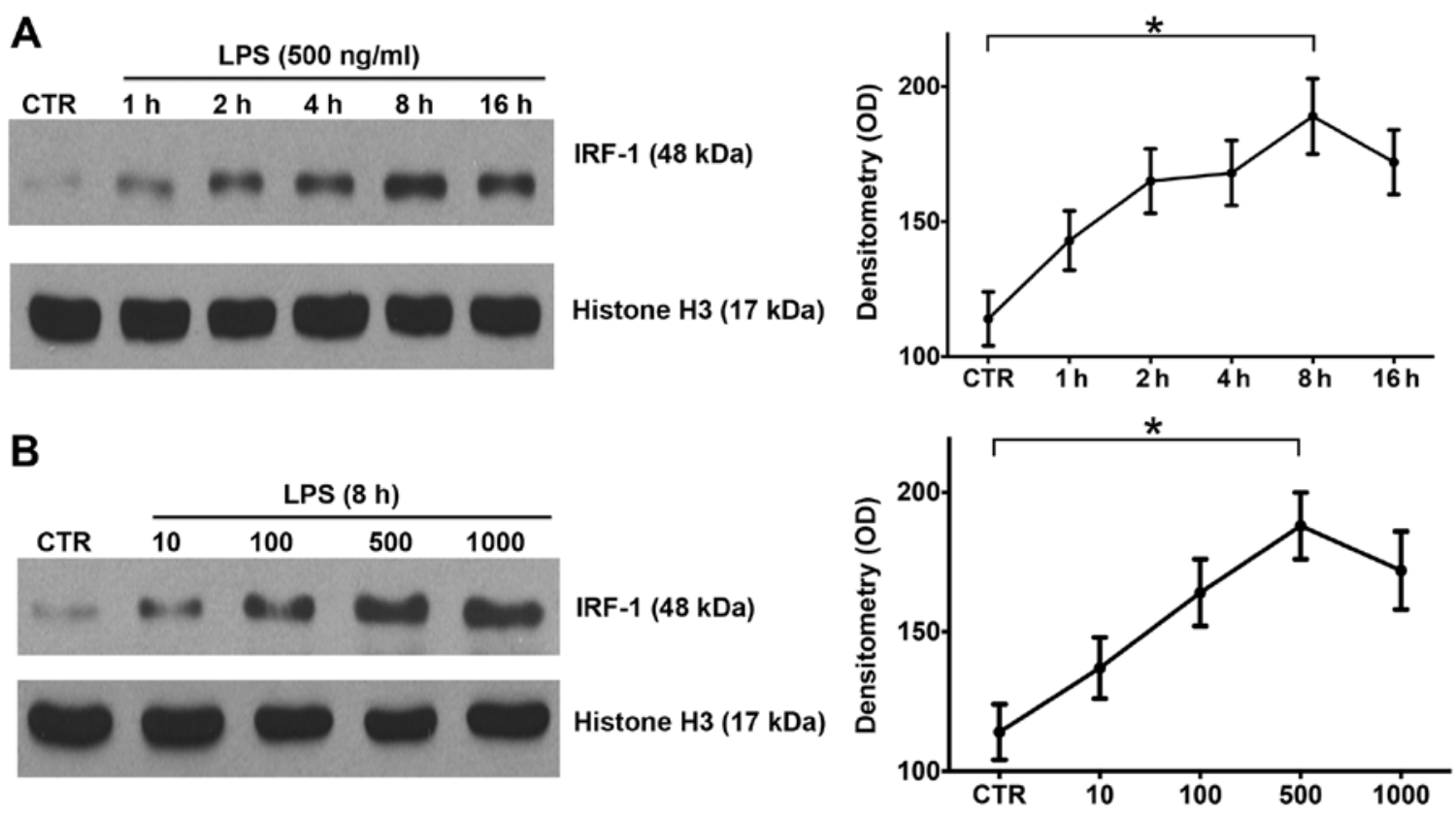

Figure 1. Lipopolysaccharide (LPS) induces interferon regulatory factor-1 (IRF-1) activation in a time- and dose-dependent manner in RAW264.7 cells (A) Following phosphate-buffered saline (PBS) or LPS $(500 \mathrm{ng} / \mathrm{ml})$ stimulation for 1-16 h, nuclear protein was analyzed for IRF-1 activation by western blot analysis. (B) Following PBS or LPS (10-1,000 ng/ml) stimulation for $8 \mathrm{~h}$, nuclear protein was analyzed for IRF-1 activation by western blot analysis. "p $<0.05$, results are representative of 3 separate independent experiments.

stimulation in a time- (Fig. 2A) and dose-dependent (Fig. 2B) manner. LPS also induced ROS production in a time- (Fig. 2C) and dose-dependent (Fig. 2D) manner.

LPS induces ATP depletion, SOD consumption and MDA accumulation in RAW264.7 cells. As previously indicated, LPS-induced oxidative stress responses are associated with apoptosis, energy metabolism fluctuations and systemic inflammation responses, all of which contribute to septic shock and multiple organ failure (5). In this study, we found that LPS stimulation induced ATP depletion (Fig. 3A), SOD consumption (Fig. 3B) and MDA accumulation (Fig. 3C) in the RAW264.7 cells in both a time- and dose-dependent manner.

IRF-1 leads to mitochondrial damage both in vivo and in vitro. To determine the role of IRF-1 in LPS-induced mitochondrial damage and ROS production in macrophages, the effects of the stable overexpression or knockdown of IRF-1 in murine RAW264.7 macrophage cells were assessed by western blot analysis (data not shown). IRF-1 KO and WT mice were administered LPS $(20 \mathrm{mg} / \mathrm{kg}$ ) and peritoneal macrophages were evaluated for mitochondrial depolarization by flow cytometry, mitochondrial structure damage by TEM and plasma mitochondrial DNA by qPCR. Mitochondrial depolarization was exacerbated in the cells overexpressing IRF-1 (Fig. 4A) and was attenuated in the cells in which IRF-1 was knocked down (Fig. 4A). Following the LPS injection, peritoneal macrophages from the IRF-1 KO mice demonstrated decreased mitochondrial depolarization (Fig. 4B). In addition, mitochondrial structural damage was exacerbated in IRF-1overexpressing cells (Fig. 4C), and the damage was attenuated in the cells in which IRF-1 was knocked down (Fig. 4C). The LPS-induced mitochondrial structural damage in the peritoneal macrophages was also diminished in the IRF-1 KO mice (Fig. 4D). Furthermore, IRF-1 KO significantly reduced circulating mitochondrial DNA release in vivo (Fig. 4E).

IRF-1 leads to oxidative stress in macrophages. Since oxidative stress is a crucial component of sepsis (22), we assessed the role of IRF-1 in LPS-induced oxidative stress by measuring the levels of ROS, ATP, SOD and MDA. ROS production was increased in the IRF-1-overexpressing cells and was reduced in the cells which IRF-1 was knocked down (Fig. 5A). In addition, LPS-induced ROS production in the peritoneal macrophages was significantly decreased in the IRF-1 KO mice (Fig. 5B). IRF-1 overexpression further enhanced ATP depletion, while IRF-1 knockdown attenuated ATP depletion (Fig. 5C). Furthermore, although SOD consumption was reduced in the cells in which IRF-1 was knocked down, there was no apparent difference in the IRF-1-overexpressing cells (Fig. 5D). By contrast, IRF-1 overexpression increased the LPS-induced MDA accumulation; however, the difference in the cells in which IRF-1 was knocked down was not significant (Fig. 5E).

\section{Discussion}

Despite improvements in antibiotic therapies and critical care techniques, sepsis remains a fatal syndrome due to the lack of effective and targeted interventions. The life-threatening effects of sepsis involve excessive formation of inflammatory cytokines, ROS and reactive nitrogen species (RNS), which lead to oxidative stress responses, mitochondrial dysfunction and organ failure $(2,23)$. Since the mitochondria regulate vital cellular functions, including energy production, ROS and RNS generation, intrinsic apoptosis, stress and metabolic signals transduction, they are important to the pathogenesis of sepsis (24-26). Thus, 

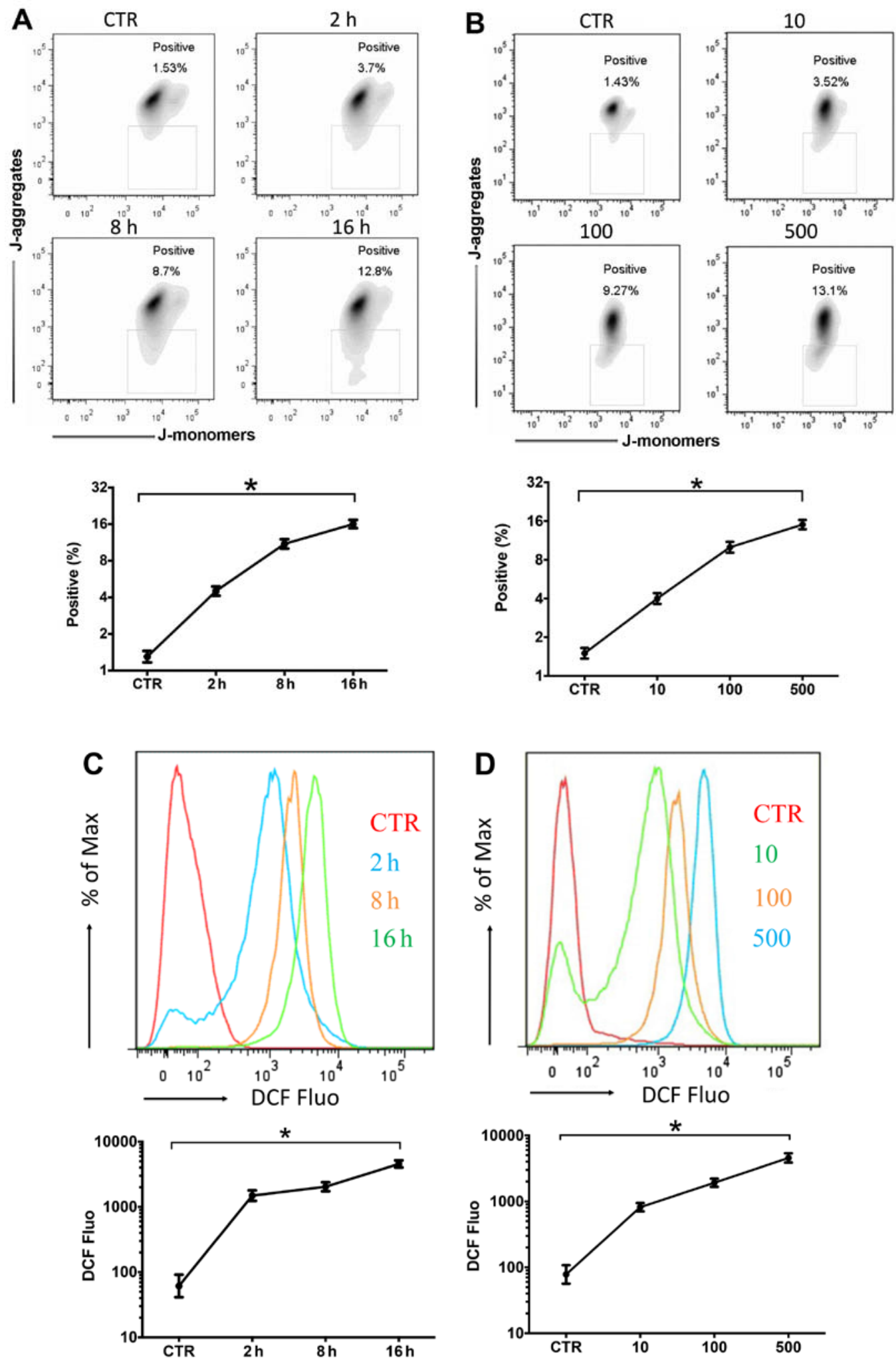

Figure 2. Lipopolysaccharide (LPS) induces mitochondrial damage and reactive oxygen species (ROS) production in a time- and dose-dependent manner in RAW264.7 cells. (A) Following phosphate-buffered saline (PBS) or LPS (500 ng/ml) stimulation for 2-16 h, cells were analyzed by flow cytometry for JC-1. (B) Following PBS or LPS (10-500 ng/ml) stimulation for $16 \mathrm{~h}$, cells were analyzed by flow cytometry for JC-1. (C) Following PBS or LPS (500 ng/ml) stimulation for 2-16 h, cells were analyzed by flow cytometry for 2,7-dichlorofluorescin diacetate (DCFH-DA). (D) Following PBS or LPS (10-500 ng/ml) stimulation for $16 \mathrm{~h}$, cells were analyzed by flow cytometry for DCFH-DA. " $\mathrm{p}<0.05$, results are representative of 3 separate independent experiments.

attenuating mitochondrial damage and oxidative stress responses may be promising therapeutic targets for the treatment of sepsis.

Mitochondrial structural damage and dysfunction has been recognized as an important molecular pathology in sepsis and is linked to the severity of organ dysfunction and outcome of sepsis (27). Peripheral blood mononuclear cells in septic patients have significant mitochondrial damage, and this is associated with the abundance of inflammatory cytokines. For patients 

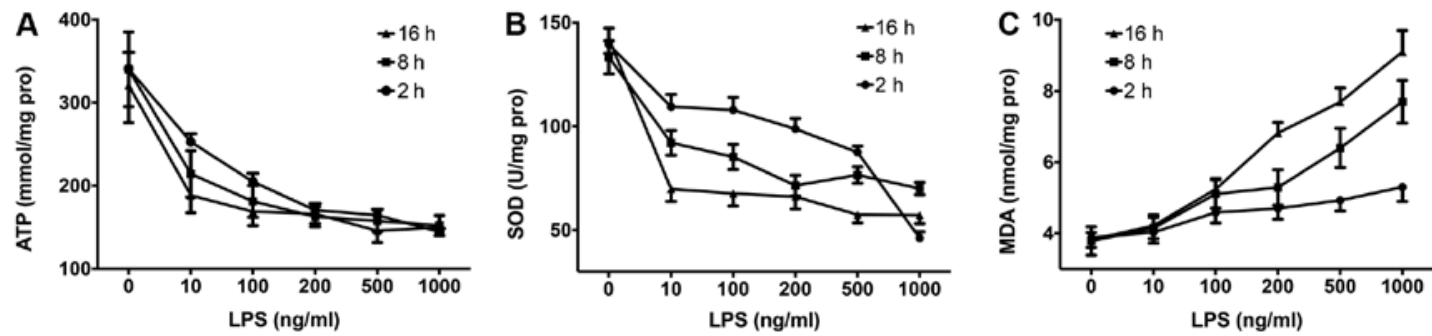

Figure 3. Lipopolysaccharide (LPS) induces adenosine triphosphate (ATP) depletion, superoxide dismutase (SOD) consumption, and malondialdehyde (MDA) accumulation in a time- and dose-dependent manner in RAW264.7 cells. The cells were stimulated with LPS (10-1,000 ng/ml) for 2-16 h. Intracellular (A) ATP, (B) SOD and (C) MDA levels were assayed using commercial kits. Results are representative of 3 separate and independent experiments.
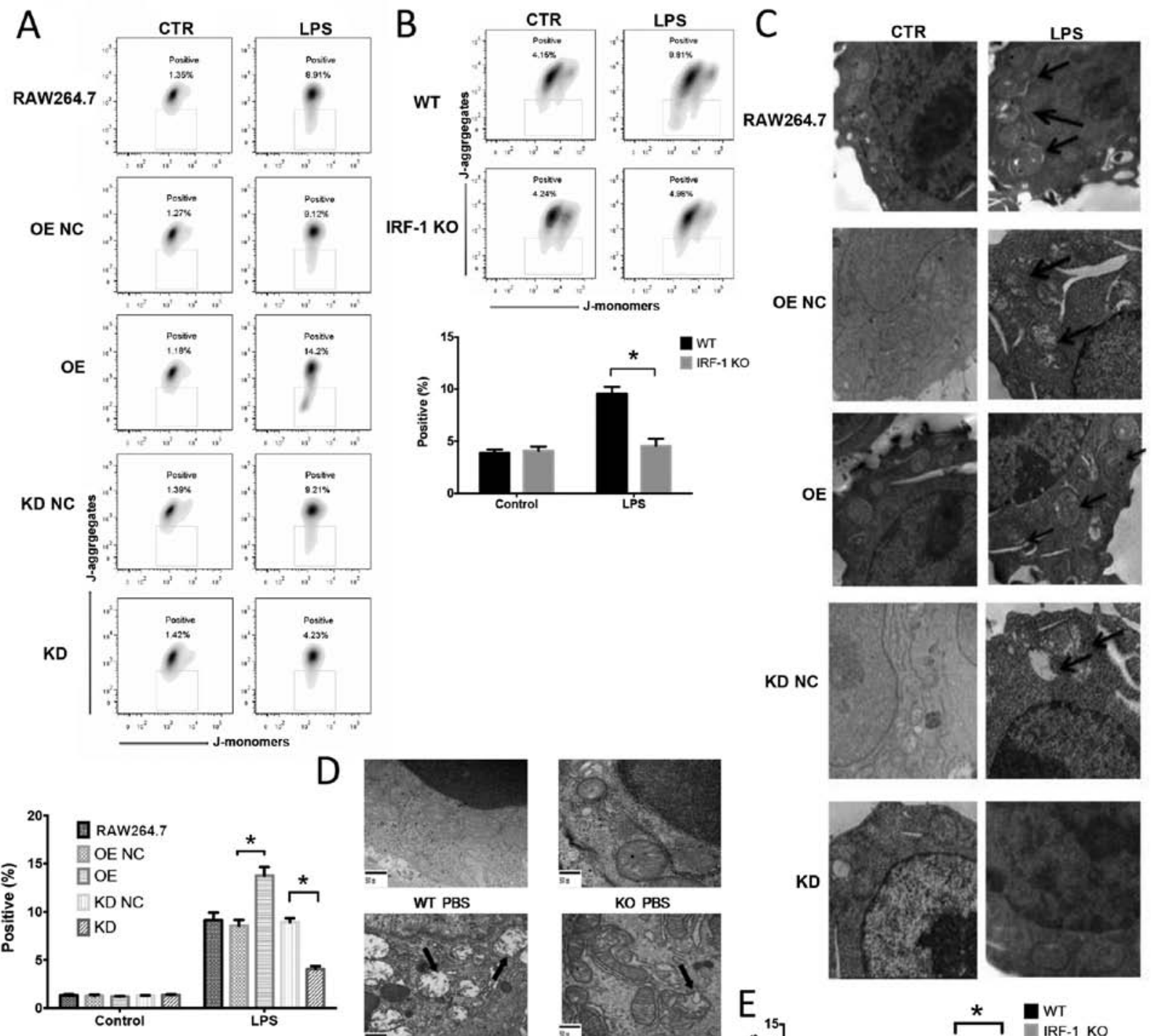

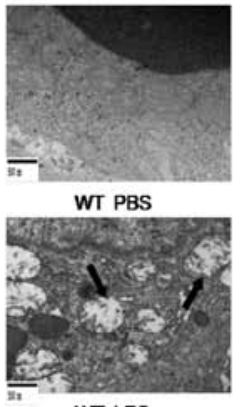

WT LPS

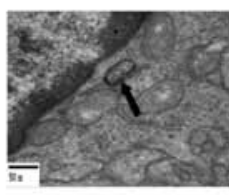

KO LPS

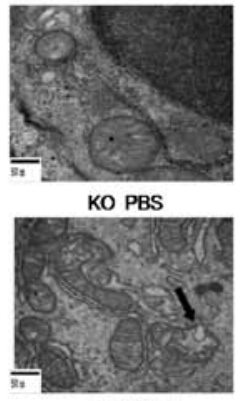

WT LPS

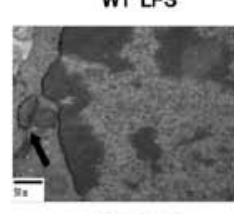

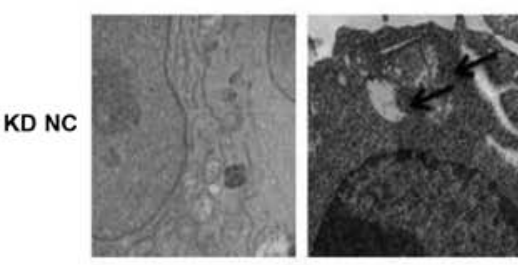
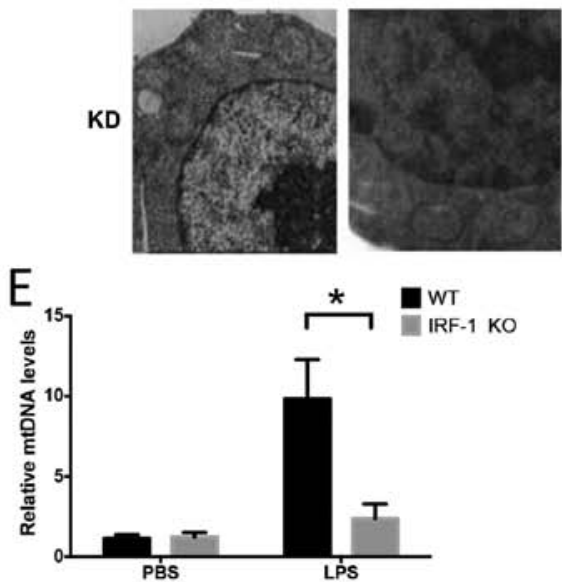

Figure 4. Interferon regulatory factor-1 (IRF-1) leads to mitochondrial structure damage both in vivo and in vitro. RAW264.7 cells were transfected with IRF-1 shRNA, control lentivirus vector (NC shRNA), IRF-1 overexpression lentivirus (IRF-1 OE), or control lentivirus vector (OE NC). RAW264.7 cells were then stimulated with phosphate-buffered saline (PBS) or lipopolysaccharide (LPS) (100 ng/ml) for $16 \mathrm{~h}$. (A) Mitochondrial transmembrane potential was assessed using JC-1 by flow cytometry. (B) IRF-1 KO and wild-type (WT) mice were administered LPS (20 mg/kg) by intraperitoneal (IP) injection; Peritoneal macrophages were later isolated and detected similarly with RAW264.7 cells. (C and D) Furthermore, cells were imaged by transmission electron microscope for mitochondrial structure damage (magnification, $\mathrm{x} 20,000$ ). (E) The plasma mitochondrial DNA (mtDNA) level was measured by $\mathrm{qPCR}$. ${ }^{*} \mathrm{p}<0.05$, results are representative of 3 separate and independent experiments. Arrows indicate damaged mitochondria. 

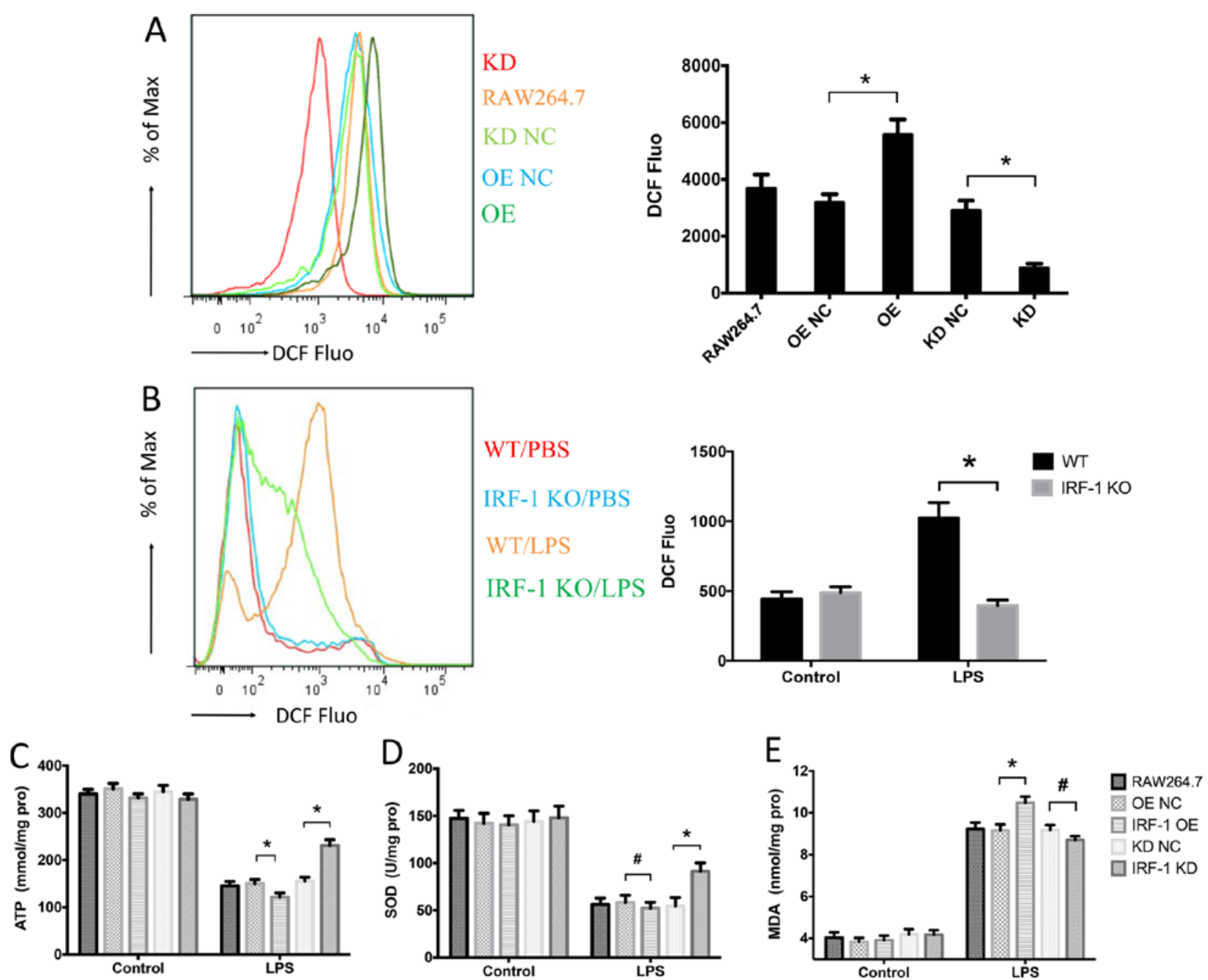

Figure 5. Interferon regulatory factor-1 (IRF-1) leads to oxidative stress in macrophages. RAW264.7 cells were transfected with IRF-1 shRNA, control lentivirus vector (NC shRNA), IRF-1 overexpression lentivirus (IRF-1 OE) and control lentivirus vector (OE NC). Then RAW264.7 cells were then stimulated with phosphate-buffered saline (PBS) or lipopolysaccharide (LPS) $(500 \mathrm{ng} / \mathrm{ml}$ ) for $16 \mathrm{~h}$. (A) Reactive oxygen species (ROS) production was detected by flow cytometry. (B) IRF-1 KO and wild-type (WT) mice were treated with PBS or LPS (20 mg/kg) for $16 \mathrm{~h}$. Peritoneal macrophages were isolated as previously described. ROS were detected by flow cytometer. Furthermore, RAW264.7 cells were stimulated with PBS or LPS (500 ng/ml) for $16 \mathrm{~h}$. Intracellular (C) ATP, (D) superoxide dismutase (SOD), and (E) malondialdehyde (MDA) levels were assayed using commercial kits. ${ }^{*} \mathrm{p}<0.05$ and ${ }^{\#} \mathrm{p}>0.05$, results are representative of 3 separate and independent experiments.

with severe sepsis, mitochondrial functional deficiency is associated with clinical outcomes (28). Macrophage mitochondrial damage is involved in the pathogenesis of sepsis through several mechanisms (5). Specifically, mitochondrial damage-induced intrinsic apoptotic pathways cause macrophage apoptosis, leading to immunosuppression (29). In addition, damaged mitochondria release mtDNA, which join forces with ROS, and also activates NALP3 IL-1 and IL-18, leading to caspase-1 processing and maturation in macrophages $(30,31)$. Thus, the upstream signaling that mediates macrophage mitochondrial dysfunction may be a potential therapeutic target.

Mitochondria are membrane-bound organelles that maintain cellular energy production through oxidative phosphorylation. As the mitochondria are prone to damage during stress, mitochondrial dynamics are tightly controlled to maintain homeostasis (32-34). Autophagy, through the identification and removal of damaged mitochondria, is an important mechanism of mitochondrial quality control to maintain homeostasis. Defects in autophagy lead to the delayed removal and heightened accumulation of damaged mitochondria in the cytoplasm, which then triggers oxidative stress, inflammation, apoptosis and other reactions $(32,33)$. The presence of IRF-1 has been shown to be associated with less autophagy in both splenocytes and macrophages $(12,13)$.

In this study, we reported that LPS promoted IRF-1 activation in macrophages in both a time-and dose-dependent manner. IRF-1 overexpression can lead to mitochondrial structural damage and oxidative stress responses, whereas IRF-1 knockdown or knockout is associated with less mitochondrial damage and oxidative stress, which may be explained by decreased autophagic responses and increased mitochondrial damage triggering intrinsic macrophage apoptosis.

The mechanisms underlying macrophage mitochondrial damage in during sepsis involve defects in energy production, apoptosis and the induction of inflammation (5). Increased mitochondrial ROS during sepsis can inhibit oxidative phosphorylation and ATP generation, which unbalance energy metabolism and cause systemic inflammation and multiple 
organ failure $(22,35)$. ROS can subsequently impair mitochondrial structures and halt mitochondrial biogenesis (36-38). These events form a feedback loop, such that targeting IRF-1 may inhibit macrophage mitochondrial damage and ROS production, alleviate oxidative stress, and in turn improve endotoxemia outcomes.

A recent study implicated the mitochondria in the regulation of inflammation and mitochondrial derived danger-associated molecular patterns (DAMPs), including ROS, mtDNA, $\mathrm{N}$-formyl peptides and cytochrome $c$ (39). During sepsis, damaged mitochondrial release mitochondrial DNA, a type of DAMP, through the TLR9 receptor, activating the MAPK signal transduction pathway. Following the tail vein injection of LPS, mitochondrial fragments containing mtDNA can be found to directly cause systemic inflammatory responses, acute lung injury, and neutrophil infiltration in the liver and kidney of the mice $(39,40)$. In this study, we confirmed that circulating mtDNA release was decreased in IRF-1 KO mice; however, the role of mtDNA in IRF-1-induced systemic inflammatory responses remains unclear. Although the hallmarks of mitochondrial dysfunction, including oxidative stress and impaired ATP production, are evident in macrophages during endotoxemia, whether these processes cause or are a result of systemic inflammation is unknown. To study mitochondrial function in the setting of overwhelming systemic inflammation during endotoxemia may elucidate these specifics. We confirmed that IRF-1 is important to LPS-induced mitochondrial structural damage and oxidative stress response in macrophages and suggest that IRF-1 may be a potential therapeutic target in the treatment of endotoxemia.

\section{Acknowledgements}

This study was supported by the National Natural Science Foundation of China (no. 81401631) and the Health and Family Planning Commission of Hunan Province (no. 14JJ7010).

\section{References}

1. Aneja R and Fink MP: Promising therapeutic agents for sepsis. Trends Microbiol 15: 31-37, 2007.

2. Stearns-Kurosawa DJ, Osuchowski MF, Valentine C, Kurosawa S and Remick DG: The pathogenesis of sepsis. Annu Rev Pathol 6: 19-48, 2011.

3. Cheng B, Xie G, Yao S, Wu X, Guo Q, Gu M, Fang Q, Xu Q, Wang D, Jin Y, et al: Epidemiology of severe sepsis in critically ill surgical patients in ten university hospitals in China. Crit Care Med 35: 2538-2546, 2007.

4. Park BS, Song DH, Kim HM, Choi BS, Lee H and Lee JO: The structural basis of lipopolysaccharide recognition by the TLR4-MD-2 complex. Nature 458: 1191-1195, 2009.

5. Singer M: The role of mitochondrial dysfunction in sepsisinduced multi-organ failure. Virulence 5: 66-72, 2014.

6. Mège JL, Mehraj V and Capo C: Macrophage polarization and bacterial infections. Curr Opin Infect Dis 24: 230-234, 2011.

7. Garrabou G, Morén C, López S, Tobías E, Cardellach F, Miró O and Casademont J: The effects of sepsis on mitochondria. J Infect Dis 205: 392-400, 2012

8. Chen W and Royer WE Jr: Structural insights into interferon regulatory factor activation. Cell Signal 22: 883-887, 2010.

9. Tamura T, Yanai H, Savitsky D and Taniguchi T: The IRF family transcription factors in immunity and oncogenesis. Annu Rev Immunol 26: 535-584, 2008.

10. Pan PH, Cardinal J, Li ML, Hu CP and Tsung A: Interferon regulatory factor- 1 mediates the release of high mobility group box-1 in endotoxemia in mice. Chin Med J (Engl) 126: 918-924, 2013.
11. Senaldi G, Shaklee CL, Guo J, Martin L, Boone T, Mak TW and Ulich TR: Protection against the mortality associated with disease models mediated by TNF and IFN-gamma in mice lacking IFN regulatory factor-1. J Immunol 163: 6820-6826, 1999.

12. Zhang L, Cardinal JS, Bahar R, Evankovich J, Huang H, Nace G, Billiar TR, Rosengart MR, Pan P and Tsung A: Interferon regulatory factor-1 regulates the autophagic response in LPS-stimulated macrophages through nitric oxide. Mol Med 18: 201-208, 2012.

13. Zhang L, Cardinal JS, Pan P, Rosborough BR, Chang Y, Yan W, Huang H, Billiar TR, Rosengart MR and Tsung A: Splenocyte apoptosis and autophagy is mediated by interferon regulatory factor 1 during murine endotoxemia. Shock 37: 511-517, 2012.

14. Chen F, Chen B, Xiao FQ, Wu YT, Wang RH, Sun ZW, Fu GS, Mou Y, Tao W, Hu XS, et al: Autophagy protects against senescence and apoptosis via the RAS-mitochondria in highglucose-induced endothelial cells. Cell Physiol Biochem 33: 1058-1074, 2014.

15. Chen S, Huang J, Zeng Q, Jia Y and Wang J: Effect of autophagy and mitochondrial coenzyme $Q$ on exocrine function of pancreas in rats with acute sepsis. Zhonghua Wei Zhong Bing Ji Jiu Yi Xue 27: 86-91, 2015 (In Chinese).

16. Su Y, Qu Y,Zhao F, Li H, Mu D and Li X: Regulation of autophagy by the nuclear factor $\mathrm{\kappa B}$ signaling pathway in the hippocampus of rats with sepsis. J Neuroinflammation 12: 116, 2015.

17. Gao J, Senthil M, Ren B, Yan J, Xing Q, Yu J, Zhang L and Yim JH: IRF-1 transcriptionally upregulates PUMA, which mediates the mitochondrial apoptotic pathway in IRF-1-induced apoptosis in cancer cells. Cell Death Differ 17: 699-709, 2010.

18. Lee HJ, Oh YK, Rhee M, Lim JY, Hwang JY, Park YS, Kwon Y, Choi KH, Jo I, Park SI, et al: The role of STAT1/IRF-1 on synergistic ROS production and loss of mitochondrial transmembrane potential during hepatic cell death induced by LPS/d-GalN. J Mol Biol 369: 967-984, 2007.

19. Layoun A, Samba M and Santos MM: Isolation of murine peritoneal macrophages to carry out gene expression analysis upon Toll-like receptors stimulation. J Vis Exp 98: e52749, 2015.

20. Carchman EH, Rao J, Loughran PA, Rosengart MR and Zuckerbraun BS: Heme oxygenase-1-mediated autophagy protects against hepatocyte cell death and hepatic injury from infection/sepsis in mice. Hepatology 53: 2053-2062, 2011.

21. Zheng G, Lyu J, Liu S, Huang J, Liu C, Xiang D, Xie M and Zeng Q: Silencing of uncoupling protein 2 by small interfering RNA aggravates mitochondrial dysfunction in cardiomyocytes under septic conditions. Int J Mol Med 35: 1525-1536, 2015.

22. Galley HF: Oxidative stress and mitochondrial dysfunction in sepsis. Br J Anaesth 107: 57-64, 2011.

23. Víctor VM, Espulgues JV, Hernández-Mijares A and Rocha M: Oxidative stress and mitochondrial dysfunction in sepsis: A potential therapy with mitochondria-targeted antioxidants. Infect Disord Drug Targets 9: 376-389, 2009.

24. Arulkumaran N, Deutschman CS, Pinsky MR, Zuckerbraun B, Schumacker PT, Gomez H, Gomez A, Murray P and Kellum JA; ADQI XIV Workgroup: Mitochondrial function in sepsis. Shock 45: 271-281, 2016.

25. Galluzzi L, Kepp O and Kroemer G: Mitochondria: Master regulators of danger signalling. Nat Rev Mol Cell Biol 13: 780-788, 2012.

26. Tait SW and Green DR: Mitochondria and cell signalling. J Cell Sci 125: 807-815, 2012.

27. Azevedo LC: Mitochondrial dysfunction during sepsis. Endocr Metab Immune Disord Drug Targets 10: 214-223, 2010.

28. Brealey D, Brand M, Hargreaves I, Heales S, Land J, Smolenski R, Davies NA, Cooper CE and Singer M: Association between mitochondrial dysfunction and severity and outcome of septic shock. Lancet 360: 219-223, 2002.

29. Chung CS, Song GY, Lomas J, Simms HH, Chaudry IH and Ayala A: Inhibition of Fas/Fas ligand signaling improves septic survival: Differential effects on macrophage apoptotic and functional capacity. J Leukoc Biol 74: 344-351, 2003.

30. Nakahira K, Haspel JA, Rathinam VA, Lee SJ, Dolinay T, Lam HC, Englert JA, Rabinovitch M, Cernadas M, Kim HP, et al: Autophagy proteins regulate innate immune responses by inhibiting the release of mitochondrial DNA mediated by the NALP3 inflammasome. Nat Immunol 12: 222-230, 2011.

31. Zhou R, Yazdi AS, Menu P and Tschopp J: A role for mitochondria in NLRP3 inflammasome activation. Nature 469: 221-225, 2011.

32. Green DR, Galluzzi L and Kroemer G: Mitochondria and the autophagy-inflammation-cell death axis in organismal aging. Science 333: 1109-1112, 2011. 
33. Levine B, Mizushima N and Virgin HW: Autophagy in immunity and inflammation. Nature 469: 323-335, 2011.

34. Youle RJ and Narendra DP: Mechanisms of mitophagy. Nat Rev Mol Cell Biol 12: 9-14, 2011.

35. Andrades M, Ritter C, de Oliveira MR, Streck EL, Fonseca Moreira JC and Dal-Pizzol F: Antioxidant treatment reverses organ failure in rat model of sepsis: Role of antioxidant enzymes imbalance, neutrophil infiltration, and oxidative stress. J Surg Res 167: e307-e313, 2011.

36. Shokolenko I, Venediktova N, Bochkareva A, Wilson GL and Alexeyev MF: Oxidative stress induces degradation of mitochondrial DNA. Nucleic Acids Res 37: 2539-2548, 2009.

37. Tsutsui H, Kinugawa S and Matsushima S: Oxidative stress and mitochondrial DNA damage in heart failure. Circ J 72 (Suppl A): A31-A37, 2008.
38. Zhang Q, Itagaki $\mathrm{K}$ and Hauser CJ: Mitochondrial DNA is released by shock and activates neutrophils via p38 map kinase. Shock 34: 55-59, 2010.

39. Zhang Q, Raoof M, Chen Y, Sumi Y, Sursal T, Junger W, Brohi K, Itagaki K and Hauser CJ: Circulating mitochondrial DAMPs cause inflammatory responses to injury. Nature 464: 104-107, 2010.

40. Zhang Z, Shi L, Song L, Ephrem E, Petri M and Sullivan KE: Interferon regulatory factor 1 marks activated genes and can induce target gene expression in systemic lupus erythematosus. Arthritis Rheumatol 67: 785-796, 2015. 